\title{
QUALIDADE FÍSICA DO SOLO EM UM LATOSSOLO VERMELHO DA REGIÃO SUDOESTE DOS CERRADOS SOB DIFERENTES SISTEMAS DE USO E MANEJO(1)
}

\author{
José Antônio Maior Bono ${ }^{(2)}$, Manuel Cláudio Motta Macedo $^{(3)}$ \& Cássio Antônio \\ Tormena $^{(4)}$
}

\begin{abstract}
RESUMO
A busca de sistemas de manejo do solo como aqueles que integram lavoura e pecuária, visando à recuperação e, ou, manutenção da qualidade do solo, é fundamental para o manejo sustentável da agropecuária. A hipótese deste estudo é que o uso do solo em sistema de integração lavoura-pecuária melhora as suas propriedades físicas, contribuindo dessa maneira para uma exploração agrícola sustentável do solo na região sudoeste do Cerrado. $O$ objetivo deste trabalho foi verificar a influência de diferentes sistemas de manejo em algumas propriedades físicas de um solo na região dos Cerrados. O solo é classificado como Latossolo Vermelho distrófico, na região do Cerrado, onde foram avaliados os seguintes sistemas de manejo: solo sob vegetação natural; solo com implantação de lavoura de soja durante um ano e seguido de três anos de pastagens; solo com implantação de lavoura de soja durante quatro anos, seguidos por quatro anos de pastagem; solo com lavoura de soja contínua em sistema de plantio direto; e solo com pastagem continuamente. Foram avaliadas a densidade do solo, a resistência do solo à penetração e a estabilidade dos agregados em água, no período de 1995 a 2006.0 uso do solo com a forrageira Brachiaria decumbens como pastagem contínua proporcionou menor impacto nas propriedades físicas do solo, em relação aos manejos com integração lavoura-pecuária e lavoura contínua.
\end{abstract}

Termos de indexação: integração lavoura-pecuária, física do solo, uso do solo, manejo do solo.

\footnotetext{
(1) Parte da Tese de Doutorado do primeiro autor apresentada ao Programa de Pós-Graduação em Solos e Nutrição de Plantas da Universidade Estadual de Maringá. Recebido para publicação em 10 de fevereiro de 2012 e aprovado em 7 de março de 2013.

(2) Professor Titular da Universidade Anhanguera - Uniderp, Unidade Agrárias. Rua Alexandre Herculano, 1400. Jardim Veraneio. CEP 79037-280 Campo Grande (MS). E-mail: bono@uniderp.edu.br

(3) Pesquisador da Embrapa Gado de Corte. BR 262, km 04. CEP 79002-970 Campo Grande (MS). Bolsista do CNPq. E-mail: manuel.macedo@embrapa.br

(4) Professor Associado do Departamento de Agronomia da Universidade Estadual de Maringá - UEM. Av. Colombo, 5790. CEP 87020-900 Maringá (PR). E-mail: catormena@uem.br
} 


\title{
SUMMARY: SOIL PHYSICAL QUALITY OF AN OXISOIL IN THE SOUTH- WESTERN CERRADO REGION UNDER DIFFERENT USE AND MANAGEMENT SYSTEMS
}

\begin{abstract}
Soil management systems, as those that integrate crop and livestock production, aiming at maintenance or recovering of soil quality, are indispensable for a sustainable agricultural management. Our hypothesis is that the land use for integrated crop-livestock systems improves soil physical properties, thus contributing to a sustainable agricultural soil use in the southwestern Cerrado region. The aim of this study was to investigate the influence of different tillage systems on some soil physical properties of the Cerrado. The soil under study was classified as an Oxisol and the following management systems were evaluated: a) soil under natural vegetation; b) soil under soybean for one year followed by three years of pasture; c) soil under soybean for four years, followed by four years of pasture; d) soil under continuous notillage soybean; e) soil under continuous pasture. Soil density, soil penetration resistance and aggregate stability in water were evaluated from 1995-2006. The soil use for continuous Brachiaria decumbens pasture degraded the soil physical properties less than crop-livestock integration and continuous crop systems.
\end{abstract}

Index terms: crop-livestock integration, soil physics, soil use, soil management.

\section{INTRODUÇÃO}

A exploração da agropecuária em solos de Cerrado tem apresentado ruptura na sustentabilidade dos recursos desse ecossistema, em face da deterioração física do solo (Costa et al., 2002; Kluthcouski \& Stone, 2003), podendo em alguns casos comprometer a qualidade das águas dos mananciais (Lal, 1998).

Assim, a busca da qualidade do solo, identificando as limitações que esse oferece ao crescimento das plantas, é um dos principais objetivos das pesquisas em física do solo, como demonstraram trabalhos de Kay (1990), Tormena et al. (1998) e Silva et al. (2001). Andrews et al. (2002), sem especificar nenhuma propriedade física do solo, destacaram que a qualidade do solo provém dos componentes da qualidade do ambiente, influenciando a produtividade e contribuindo para a degradação do agrossistema.

A qualidade física do solo foi definida por Kay et al. (1994) como o crescimento das plantas sendo influenciado pelas propriedades do solo, como porosidade total, densidade, compactação relativa, distribuição dos tamanhos dos agregados e disponibilidade de água. Hussain et al. (1997) consideraram porosidade, densidade do solo e estabilidade de agregados como indicadores dessa qualidade.

Marchão et al. (2007), trabalhando com a integração lavoura-pecuária no Cerrado, verificaram que esse sistema até a camada de $5 \mathrm{~cm}$ de profundidade causa alteração na qualidade físico-hídrico do solo, quando comparado à vegetação natural.

Lima et al. (2007), trabalhando com a densidade do solo e a produção de forragem de milho, verificaram que essa propriedade do solo é indicador da sua qualidade física. Alves et al. (2007) também verificaram que a densidade do solo favoreceu a qualidade dele, além de aumentar a infiltração de água nele.

A maioria das avaliações das propriedades físicas do solo, como índice de qualidade, é realizada em determinados momentos, não considerando sua dinâmica ao longo do tempo. Os acompanhamentos temporais dessas propriedades e em diferentes sistemas de manejo podem determinar, de maneira mais conclusiva, a importância dessas propriedades na avaliação da qualidade dos solos.

A hipótese deste trabalho é que o manejo do solo em sistema de integração lavoura-pecuária melhora as suas propriedades físicas, contribuindo para uma exploração agrícola mais sustentável do recurso natural solo, na região do Cerrado.

O objetivo deste trabalho foi verificar a influência de diferentes sistemas de manejo na qualidade física de um Latossolo Vermelho distrófico, ao longo do tempo na região do Cerrado.

\section{MATERIAL E MÉTODOS}

A área utilizada para o experimento está localizada no Centro Nacional de Pesquisa de Gado de Corte Embrapa Gado de Corte, em Campo Grande, Estado de Mato Grosso do Sul, em um solo descrito como Latossolo Vermelho distrófico (Embrapa, 1999).

O local foi desmatado entre 1972-1973, onde havia vegetação típica de Cerradão (Ferri, 1977). Em outubro de 1987, foi realizada uma gradagem pesada, retirada das raízes, calagem $\left(1,0 \mathrm{t} \mathrm{ha}^{-1}, \mathrm{PRNT}=100 \%\right) \mathrm{e}$ adubação da área ( $350 \mathrm{~kg} \mathrm{ha}^{-1}$ de superfosfato simples, $100 \mathrm{~kg} \mathrm{ha}^{-1}$ de cloreto de potássio e $40 \mathrm{~kg} \mathrm{ha}^{-1}$ de FTE), incorporadas com grade niveladora. A partir daí, até o ano de 1993, o local foi utilizado pelo Centro para 
manutenção geral do rebanho. Nessa época, o solo apresentava, de acordo com os quadros 1 e 2 , as seguintes características químicas e granulométricas, respectivamente.

Entre setembro e outubro de 1993 foi efetuada uma calagem para atingir saturação por bases entre $40 \mathrm{e}$ $45 \%$, e realizada em duas etapas, sendo $2 / 3$ antes da aração e 1/3 após a aração com arado de aiveca a 0,30 $0,35 \mathrm{~m}$, conforme o sistema inverso do solo (Seguy et al., 1984). Em seguida, foi feita uma gradagem niveladora para uniformizar a área e incorporar $1 / 3$ restante do calcário.

Após a calagem, foi realizada uma adubação fosfatada ( $80 \mathrm{~kg} \mathrm{ha}^{-1}$ de $\mathrm{P}_{2} \mathrm{O}_{5}$ ), utilizando-se como fonte o superfosfato simples aplicado a lanço e incorporado com grade niveladora em toda a área experimental, com exceção de uma pequena área de pastagem em degradação, deixada como referência.

\section{Tratamentos}

Neste trabalho, foram considerados cinco tratamentos de manejo do solo: CERR - solo com vegetação natural; PCCA - solo com pastagem Brachiaria decumbens cultivar Basilisk contínua e adubada; L1P3 - solo sob lavoura de soja por um e por três anos sob pastagem com Brachiaria brizantha cultivar Marandu; L4P4 - solo sob lavoura de soja por quatro anos e por quatro anos sob pastagem com Panicum maximum cultivar Tanzânia-1; e LCPD lavoura contínua de soja em sistema de plantio direto.

As amostragens nos tratamentos iniciaram-se a partir de julho de 1995, após dois anos da sua implantação.

\section{Descrição dos tratamentos}

No tratamento PCCA, a implantação da pastagem Brachiaria decumbens cv. Basilisk foi feita em

Quadro 1. Características químicas do solo da área em estudo antes da implantação do experimento, nas profundidades avaliadas

\begin{tabular}{|c|c|c|}
\hline \multirow{2}{*}{ Característica $^{(1)}$} & \multicolumn{2}{|c|}{ Profundidade $(\mathrm{cm})$} \\
\hline & $0,0-0,20$ & $0,20-0,40$ \\
\hline $\mathrm{pH}\left(\mathrm{H}_{2} \mathrm{O}\right)$ & 5,14 & 5,02 \\
\hline $\mathrm{pH}\left(\mathrm{CaCl}_{2}\right)$ & 4,11 & 4,32 \\
\hline $\mathrm{P}$ disponível $\left(\mathrm{mg} \mathrm{dm}{ }^{-3}\right)$ & 1,50 & 1,00 \\
\hline $\mathrm{K}$ disponível (mg dm$\left.{ }^{-3}\right)$ & 43 & 25 \\
\hline $\mathrm{Ca}^{2+}\left(\mathrm{cmol}_{\mathrm{c}} \mathrm{dm}^{-3}\right)$ & 0,55 & 0,34 \\
\hline $\mathrm{Mg}^{2+}\left(\mathrm{cmol}_{\mathrm{c}} \mathrm{dm}^{-3}\right)$ & 0,27 & 0,19 \\
\hline $\mathrm{Al}^{3+}\left(\mathrm{cmol}_{\mathrm{c}} \mathrm{dm}^{-3}\right)$ & 0,95 & 1,10 \\
\hline $\mathrm{H}+\mathrm{Al}\left(\mathrm{cmol}_{\mathrm{c}} \mathrm{dm}^{-3}\right)$ & 7,24 & 6,55 \\
\hline Matéria orgânica $\left(\mathrm{g} \mathrm{dm}^{-3}\right)$ & 32,0 & 21,0 \\
\hline Saturação por bases (\%) & 8,2 & 8,3 \\
\hline
\end{tabular}

(1) Métodos descritos em Embrapa (1997). parcelas com dimensões de 140 x $50 \mathrm{~m}\left(7.000 \mathrm{~m}^{2}\right)$ e adubada a cada dois anos. As adubações utilizadas equivaleram ao uso de $400 \mathrm{~kg} \mathrm{ha}^{-1}$ do formulado 0-2020 , aplicado a lanço, e realizadas nos meses de novembro; a partir de 2000/2001, a pastagem passou a receber anualmente $50 \mathrm{~kg} \mathrm{ha}^{-1}$ de $\mathrm{N}$, tendo como fonte a ureia, sendo realizada entre dezembro ejaneiro.

No tratamento com lavoura contínua, em sistema de plantio direto (LCPD), com parcelas com dimensões de $110 \times 12 \mathrm{~m}\left(1.320 \mathrm{~m}^{2}\right)$, o preparo inicial foi feito como descrito anteriormente. No ano agrícola de 1994/ 95, iniciou-se o plantio de soja como cultura de verão, não ocorrendo mais o preparo do solo. A adubação do solo era feita na cultura de verão, utilizando-se 400 a $450 \mathrm{~kg} \mathrm{ha}^{-1}$ do formulado 00-20-20. No cultivo de inverno, utilizou-se milheto e sorgo, sendo que a partir de 2000/2001, passou-se a semear sorgo consorciado com braquiária para incrementar a formação de palhada. Na área, foi efetuada aplicação superficial de calcário nos anos de 1998 e 2002, para manter saturação por bases entre $45-50 \%$. A média da produtividade de soja, no período estudado ficou em $2.435 \mathrm{~kg} \mathrm{ha}^{-1}$.

No tratamento L1P3, utilizaram-se parcelas de 140 x $50 \mathrm{~m}$, com um ano de lavoura de soja seguido por três anos de pastagem. Com exceção do ano de 1993, em que a lavoura de soja foi introduzida com o preparo convencional do solo, o plantio da soja foi realizado sem o preparo do solo (plantio direto), assim como o plantio da forrageira. A forrageira utilizada nesse sistema foi a Brachiaria brizantha cv. Marandu. A soja recebeu adubação de acordo com recomendação de Embrapa (1994), a partir da análise do solo. $\mathrm{Na}$ pastagem, foi aplicada adubação anual de manutenção a partir de 2002/2003 com $200 \mathrm{~kg} \mathrm{ha}^{-1}$ da fórmula 020-20 e $80 \mathrm{~kg} \mathrm{ha}^{-1} \mathrm{ano}^{-1} \mathrm{de} \mathrm{N}$, utilizando a ureia como fonte. Entre 1994 e 2001 a pastagem após soja era manejada apenas com o efeito residual da adubação da cultura. A calagem também foi realizada para manter a saturação por bases entre $45-50 \%$ nos anos de 1997 e 2003, na dose de $1.500 \mathrm{~kg} \mathrm{ha}^{-1}$. A partir de 1997, o manejo do solo foi em sistema de plantio direto, tanto da lavoura como da pastagem. A média da produtividade de soja, no período estudado, ficou em $2.520 \mathrm{~kg} \mathrm{ha}^{-1}$.

No tratamento L4P4, as parcelas foram de $140 \mathrm{x}$ $50 \mathrm{~m}\left(7.000 \mathrm{~m}^{2}\right)$. A área foi utilizada com a cultura da soja por quatro anos, seguidos por quatro anos de pastagem, utilizando-se a forrageira Panicum maximum cv. Tanzânia-1. No período de 1993 até 1996, o plantio da cultura da soja foi feito em solo preparado com grade e, a partir de 1996, sem o preparo (plantio direto). A cultura da soja recebeu adubação de acordo com recomendação de Embrapa (1994), conforme análise de solo. A pastagem recebeu adubação anual de manutenção, a partir de 2001/2002, foi composta de $200 \mathrm{~kg} \mathrm{ha}^{-1}$ da fórmula 0-20-20 e $100 \mathrm{~kg}$ $\mathrm{ha}^{-1} \mathrm{ano}^{-1}$ de N, utilizando a ureia como fonte. Entre 1994 e 2001 a pastagem após soja era manejada 
apenas com o efeito residual da adubação da cultura. A calagem também foi realizada para manter a saturação por bases entre 45-50 \% nos anos de 1997 e 2003 , na dose de $1.500 \mathrm{~kg} \mathrm{ha}^{-1}$. A média da produtividade de soja, no período estudado ficou em $2.705 \mathrm{~kg} \mathrm{ha}^{-1}$.

No tratamento CERR, consideraram-se parcelas de $140 \times 50 \mathrm{~m}\left(7.000 \mathrm{~m}^{2}\right)$ de vegetação natural existente ao lado do experimento.

\section{Delineamento estatístico}

O delineamento estatístico foi o de blocos ao acaso,istribuídos em parcelas experimentais num delineamento de blocos inteiramente casualizados com quatro repetições, sendo os anos considerados como épocas de avaliações, constituindo-se como subparcelas de um arranjo de parcelas subdivididas, para as variáveis estudadas.

\section{Implantação e manejo das forrageiras}

As pastagens utilizadas foram a Brachiaria decumbens cv. Basilisk, Brachairia brizantha cv. Marandu e Panicum maximum cv. Tanzânia-1. A semeadura das gramíneas foi feita a lanço, nas quantidades de 1,8; 3,0; e 2,0 $\mathrm{kg} \mathrm{ha}^{-1}$ de sementes puras viáveis, respectivamente para Brachiaria decumbens, Brachaira brizantha e Panicum maximum.

As forrageiras foram pastejadas em sistema contínuo, com uma taxa de lotação variável, de acordo com a disponibilidade de forragem, pré-estabelecida entre 2.500 e $3.000 \mathrm{~kg} \mathrm{ha}^{-1}$ de matéria seca total, utilizando, como categoria animal, bezerras da raça Nelore com peso vivo entre 200 e $220 \mathrm{~kg}$ por animal. A carga animal média anual para as áreas com Brachiaria decumbens e Brachiaria brizantha, durante o período estudado, ficou em torno de 1,60 unidade animal (UA). Para as áreas com Panicum maximum, a carga média anual foi de 1,70 UA.

\section{Atributos do solo avaliados}

Em dezembro de 1996, 1999, 2000, 2001 e 2002, foram coletadas amostras indeformadas em quatro locais dentro de cada parcela, utilizando-se anéis metálicos de capacidade de $0,0001 \mathrm{~m}^{3}$, em quatro profundidades: $0-0,1 ; 0,1-0,2 ; 0,2-0,3 ; \mathrm{e} 0,3-0,4 \mathrm{~m}$. Os anéis foram introduzidos no solo por percussão, utilizando um amostrador para determinar a densidade do solo (Ds) pelo método do anel volumétrico e a densidade de particulas $(\mathrm{Dp})$, pelo método do balão volumétrico, conforme Embrapa (1997).

Em dezembro de 1995, novembro de 2000 e fevereiro de 2006 , foram coletadas amostras de solo em quatro locais nas camadas de 0-0,10 e 0,10-0,20 m. As amostras foram passadas em peneira com $8 \mathrm{~mm}$ de abertura de malha, após secar ao ar e coletadas na peneira de $2 \mathrm{~mm}$, conforme Guedes et al. (1996). Em seguida, procedeu-se à análise de estabilidade de agregados, em peneiramento por via úmida, pelo método proposto por Yoder, modificado por Kemper \& Chepil (1965). Utilizaram-se $100 \mathrm{~g}$ de agregados seco ao ar, umedecidos por capilaridade, colocando-os na parte superior de um conjunto de cinco peneiras com diâmetro de abertura da malha de 2,$00 ; 1,00 ; 0,50$; 0,25 ; e $0,105 \mathrm{~mm}$ e agitando-as verticalmente com 46 oscilações verticais por minuto dentro da água, durante $10 \mathrm{~min}$. Nas amostras dos agregados secos ao ar, determinou-se a umidade pelo método gravimétrico (Embrapa, 1997), para corrigir a massa nos agregados submetidos ao tamisamento. Em razão dos diâmetros de malha das peneiras utilizadas, os agregados foram distribuídos nas seguintes classes de diâmetro equivalente: 8 - 2 ; $2-1$; $1-05 ; 0,5-0,25$; e 0,250,105 e abaixo de $0,105 \mathrm{~mm}$.

A resistência do solo à penetração (RP) foi avaliada em março de 1996, 1997, 1999, 2000, 2002 e 2006, nas camadas de 0,$05 ; 0,10 ; 0,15 ; 0,20 ; 0,25 ; 0,30 ; 0,35$; 0,$40 ;$ e $0,45 \mathrm{~m}$. Foi utilizado um penetrógrafo, modelo SC-60, munido de um cone de diâmetro da base de 0,01 m. Em cada parcela, estabeleceram-se duas linhas e 10 pontos de amostragem por linha. As linhas e os pontos ficaram equidistantes $10 \mathrm{~m}$ entre si, nos tratamentos com pastagem. Nas parcelas sob plantio direto, as linhas ficaram equidistantes $4 \mathrm{~m}$ e os pontos entre $10 \mathrm{~m}$, conforme figura 1 . Simultaneamente à avaliação da resistência, e nas mesmas profundidades, coletavam-se amostras de solo para determinar a umidade pelo método gravimétrico, de acordo com Embrapa (1997).

As medidas de RP foram feitas após chuvas acima de $30 \mathrm{~mm}$, suficientes para saturar o solo até $0,45 \mathrm{~m}$, entre 24 e $48 \mathrm{~h}$, após a precipitação. A umidade no período da medição da resistência variou entre 0,3263 e $0,1762 \mathrm{~g} \mathrm{~g}^{-1}$.

A correção dos valores de resistência e relação à umidade foi feita utilizando-se o modelo proposto por Busscher et al. (1997). Esse ajuste utiliza-se de dois modelos matemáticos (equações 1 e 2):

$$
C=a W^{b}
$$

em que $\mathrm{C}=$ resistência medida $(\mathrm{MPa}) ; \mathrm{W}=$ umidade gravimétrica; $a \mathrm{e} b$ são parâmetros do modelo ajustado.

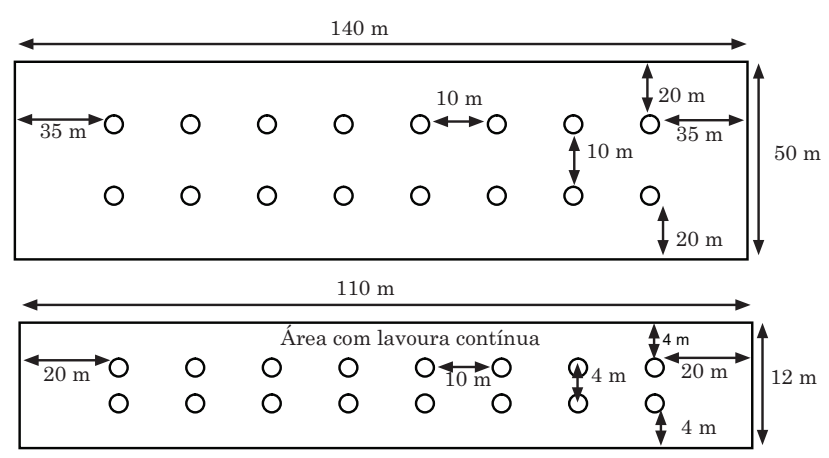

Figura 1. Esquema utilizado para determinar a resistência do solo à penetração nas áreas de pastagem e de lavoura contínua. 


$$
C c=C o+\frac{d C}{d W}(W c-W o)
$$

sendo $\mathrm{Cc}=$ resistência do solo corrigida $(\mathrm{MPa}) ; \mathrm{Co}=$ resistência do solo observada $(\mathrm{MPa})$;

$\frac{d C}{d W}=$ derivada primeira da equação 1 , em relação à umidade;

Wc = umidade para a qual os dados foram corrigidos (média da umidade de $0,226 \mathrm{~g}_{\text {água }} \mathrm{g}^{-1}$ solo);

Wo = umidade gravimétrica determinada em cada ponto de medida.

\section{Análises estatísticas}

Os valores das variáveis de densidade do solo, resistência do solo à penetração e agregados estáveis em água foram interpretados, utilizando-se a análise de variância; para comparar as médias, aplicou-se o teste de Tukey a $5 \%$, para cada profundidade, com sugerido em Vivaldi (1999).

Foi realizado estudo de correlação entre densidade do solo e resistência à penetração, conforme PimentelGomes \& Garcia (2002).

\section{RESULTADOS E DISCUSSÃO}

\section{Densidade do solo}

Os valores médios da densidade do solo (Ds) nos diferentes sistemas de manejo e períodos de avaliação são apresentados no quadro 3. Houve efeito significativo da interação entre tratamento e ano ( $p>0,01)$. O desdobramento da interação foi realizado avaliando o efeito do tratamento em cada ano em que foram feitas as medidas (Quadro 3).

Nos tratamentos L4P4 até $0,10 \mathrm{~m}$; L1P3 até 0,2 m; e LCPD até $0,30 \mathrm{~m}$ de profundidade, ocorreram alterações significativas da Ds no período estudado, em relação ao solo sob vegetação de Cerrado.

No tratamento L4P4, o aumento da Ds ao longo dos anos ocorreu na camada de 0,0-0,10 m. Esse aumento ocorreu até o ano 2000 e depois estabilizouse em $1,28 \mathrm{~kg} \mathrm{dm}^{-3}$. Nesse tratamento, mesmo com quatro anos de lavoura e mais trânsito de máquinas e implementos, a Ds média ficou abaixo de L1P3. As maiores densidades, nas camadas superficiais do solo, no L1P3, devem-se ao pisoteio animal e hábito de crescimento da forrageira $B$. brizantha, que deixa o solo mais exposto em relação às forrageiras $B$. decumbens e Tanzânia. Esses dados sugerem que o aumento da Ds atribuído ao pisoteio dos animais pode ser significativo à movimentação de tratores, máquinas e implementos na área. Souza et al. (1998) relataram que a pressão aplicada ao solo por bovinos de $400 \mathrm{~kg}$ é de $190,3 \mathrm{kPa}$, o que é bem superior a $92,1 \mathrm{kPa}$ exercida por um trator com peso de aproximadamente de $2.900 \mathrm{~kg}$.
A compactação do solo pelo pisoteio animal atingiu até a profundidade de $0,20 \mathrm{~m}$, no tratamento L1P3, superior a $0,07-0,10 \mathrm{~m}$, registrada por Silva et al. (1997). Essa compactação nos primeiros centímetros do solo também foi verificada nos trabalhos de Pinzón \& Amesquita (1991); Sheath \& Carlson (1998); Singleton \& Addison (1999) e Kondo \& Dias Júnior (1999).

Deve-se ressaltar que o tratamento PCCA, que estava sob pastejo, não influenciou a Ds. Espécies forrageiras que cobrem bem o solo, e com abundância do sistema radicular, suportam carga animal de até 1,60 UA, sem aumentar a Ds. Espécies forrageiras de hábito de crescimento prostado (B. decumbens), que proporcionam maior cobertura do solo (Kanno et al., 2004), amenizam a compactação superficial ocasionada pelo pisoteio animal.

As mudanças mais significativas foram registradas no sistema de manejo com plantio direto. $\mathrm{O}$ aumento da Ds no sistema de lavoura contínua sob plantio direto ocorreu até a camada de $0,30 \mathrm{~m}$, superando as indicações de Reichert et al. (2004), os quais registraram compactação entre 0,08 e $0,15 \mathrm{~m}$ de profundidade.

A compactação superficial do solo no sistema de plantio direto pode ser considerada normal, segundo Hakansson \& Medvedev (1995) e Silva et al. (2002), e pode não ter consequências na produtividade de culturas. Esse fato é reforçado nos dados de Macedo et al. (2004), os quais registraram que a produtividade da soja, nesse mesmo experimento e no período estudado, continuou estável em $2.585 \mathrm{~kg} \mathrm{ha}^{-1}$.

\section{Resistência do solo à penetração}

A resistência do solo à penetração $(\mathrm{RP})$ seguiu a mesma tendência da densidade, ou seja, houve interação significativa para sistema de manejo (tratamento) e ano ( $p>0,01)$. A interação foi desdobrada, analisando o efeito de ano dentro de cada tratamento (Quadro 4).

Em qualquer sistema de manejo estudado, verificou-se aumento da resistência do solo em relação ao solo com vegetação natural (CERR), independentemente da profundidade. No tratamento PCCA, independente da profundidade, não se verificou alteração significativa da resistência do solo, ao longo de 12 anos de pastagem. A $B$. decumbens utilizada como pastagem no PCCA, quando bem fertilizada e manejada, apresentou intenso crescimento e renovação radicular e, como consequência, maior teor de matéria orgânica no solo, mais resíduos vegetais sobre ele, protegendo-o, assim, da ação compressiva e cisalhante dos cascos dos animais. A influência do sistema de pastagem na qualidade física do solo também foi verificada nos trabalhos de Fassbender \& Bornemiza (1994), Silva \& Mielniczuk (1997) e Santos et al. (2005).

No tratamento L1P3, houve aumento significativo da resistência à penetração até 1997 para as camadas 0,05 e $0,10 \mathrm{~m}$ e, até 1999 , para a camada $0,15 \mathrm{~m}$. 
Quadro 3. Densidade do solo em diferentes sistemas de manejo, em razão dos anos e das profundidades

\begin{tabular}{|c|c|c|c|c|c|c|c|c|}
\hline \multirow{2}{*}{ Tratamento } & \multicolumn{5}{|c|}{ Ano } & \multirow{2}{*}{ Média } & \multicolumn{2}{|c|}{ CV } \\
\hline & 1996 & 1999 & 2000 & 2001 & 2002 & & $\mathbf{a}$ & $\mathbf{b}$ \\
\hline & \multicolumn{6}{|c|}{$\mathrm{Mg} \mathrm{m}^{-3}$} & \multicolumn{2}{|c|}{$\%$} \\
\hline \multicolumn{9}{|c|}{$0-0,10 \mathrm{~m}$} \\
\hline CERR & $1,18 \mathrm{a}$ & $1,20 \mathrm{a}$ & $1,19 \mathrm{a}$ & $1,21 \mathrm{a}$ & $1,20 \mathrm{a}$ & 1,20 & & \\
\hline PCCA & $1,25 \mathrm{a}$ & $1,26 \mathrm{a}$ & $1,24 \mathrm{a}$ & $1,22 \mathrm{a}$ & $1,23 \mathrm{a}$ & 1,24 & & \\
\hline L1P3 & $1,28 \mathrm{~b}$ & $1,33 \mathrm{a}$ & $1,34 \mathrm{a}$ & $1,33 \mathrm{a}$ & $1,32 \mathrm{ab}$ & 1,32 & 6,27 & 4,46 \\
\hline L4P4 & $1,22 \mathrm{c}$ & $1,23 \mathrm{bc}$ & $1,28 \mathrm{a}$ & $1,27 \mathrm{ab}$ & $1,29 \mathrm{a}$ & 1,26 & & \\
\hline LCPD & $1,31 \mathrm{~b}$ & $1,34 \mathrm{ab}$ & $1,36 \mathrm{a}$ & $1,35 \mathrm{ab}$ & $1,37 \mathrm{a}$ & 1,35 & & \\
\hline \multicolumn{9}{|c|}{$0,10-0,20 \mathrm{~m}$} \\
\hline CERR & $1,18 \mathrm{a}$ & $1,19 \mathrm{a}$ & $1,18 \mathrm{a}$ & $1,19 \mathrm{a}$ & $1,18 \mathrm{a}$ & 1,18 & & \\
\hline PCCA & $1,26 \mathrm{a}$ & $1,25 \mathrm{a}$ & $1,26 \mathrm{a}$ & $1,24 \mathrm{a}$ & $1,24 \mathrm{a}$ & 1,25 & & \\
\hline L1P3 & $1,25 \mathrm{~b}$ & $1,35 \mathrm{a}$ & $1,36 \mathrm{a}$ & $1,33 \mathrm{a}$ & $1,35 \mathrm{a}$ & 1,33 & 4,64 & 4,05 \\
\hline L4P4 & $1,26 \mathrm{a}$ & $1,29 \mathrm{a}$ & $1,25 \mathrm{a}$ & $1,24 \mathrm{a}$ & $1,25 \mathrm{a}$ & 1,26 & & \\
\hline LCPD & $1,30 \mathrm{~b}$ & $1,35 \mathrm{a}$ & $1,34 \mathrm{ab}$ & $1,34 \mathrm{ab}$ & $1,35 \mathrm{a}$ & 1,34 & & \\
\hline \multicolumn{9}{|c|}{$0,20-0,30 \mathrm{~m}$} \\
\hline CERR & $1,24 \mathrm{a}$ & $1,25 \mathrm{a}$ & $1,25 \mathrm{a}$ & $1,26 \mathrm{a}$ & $1,25 \mathrm{a}$ & 1,25 & & \\
\hline PCCA & $1,26 \mathrm{a}$ & $1,26 \mathrm{a}$ & $1,24 \mathrm{a}$ & $1,23 \mathrm{a}$ & $1,24 \mathrm{a}$ & 1,25 & & \\
\hline L1P3 & $1,24 \mathrm{a}$ & $1,27 \mathrm{a}$ & $1,29 \mathrm{a}$ & $1,26 \mathrm{a}$ & $1,27 \mathrm{a}$ & 1,27 & 4,68 & 3,90 \\
\hline $\mathrm{L} 4 \mathrm{P} 4$ & $1,23 \mathrm{~b}$ & $1,28 \mathrm{a}$ & $1,26 \mathrm{ab}$ & $1,24 \mathrm{~b}$ & $1,25 \mathrm{~b}$ & 1,25 & & \\
\hline LCPD & $1,27 \mathrm{~b}$ & $1,32 \mathrm{a}$ & $1,33 \mathrm{a}$ & $1,32 \mathrm{a}$ & $1,33 \mathrm{a}$ & 1,31 & & \\
\hline \multicolumn{9}{|c|}{$0,30-0,40 \mathrm{~m}$} \\
\hline CERR & $1,21 \mathrm{a}$ & $1,20 \mathrm{a}$ & $1,21 \mathrm{a}$ & $1,21 \mathrm{a}$ & $1,20 \mathrm{a}$ & 1,21 & & \\
\hline PCCA & $1,23 \mathrm{a}$ & $1,24 \mathrm{a}$ & $1,23 \mathrm{a}$ & $1,23 \mathrm{a}$ & $1,22 \mathrm{a}$ & 1,23 & & \\
\hline L1P3 & $1,23 \mathrm{a}$ & $1,22 \mathrm{a}$ & $1,24 \mathrm{a}$ & $1,24 \mathrm{a}$ & $1,23 \mathrm{a}$ & 1,23 & 4,56 & 3,81 \\
\hline L4P4 & $1,25 \mathrm{a}$ & $1,24 \mathrm{a}$ & $1,23 \mathrm{a}$ & $1,23 \mathrm{a}$ & $1,24 \mathrm{a}$ & 1,24 & & \\
\hline LCPD & $1,24 \mathrm{a}$ & $1,28 \mathrm{a}$ & $1,28 \mathrm{a}$ & $1,27 \mathrm{a}$ & $1,27 \mathrm{a}$ & 1,27 & & \\
\hline
\end{tabular}

CERR: solo com vegetação natural; PCCA: solo com pastagem contínua e adubada; L1P3: solo sob lavoura de soja por um ano e por três anos sob pastagem de Brachiaria brizantha; L4P4: solo sob lavoura por quatro anos e por quatro anos sob pastagem de Panicum maximum; e LCPD: solo sob lavoura de soja em sistema de plantio direto. CV: coeficiente de variação, (a) para subparcelas (Anos) e (b) para parcelas (Tratamento). Letras iguais na linha não diferem entre si pelo teste de Tukey a $5 \%$.

No sistema de manejo L4P4, a RP até a camada de $0,25 \mathrm{~m}$ apresentou valores inferiores nos anos de 1996 e 1997, em relação aos demais anos. A partir de 1999 , os valores de RP tiveram aumento significativo. Os menores valores de RP, nos anos de 1996 e 1997, devem-se ao preparo do solo com grade (sistema convencional). A partir de 1998 é que se iniciou o sistema de plantio direto e, como consequência, houve aumento da resistência do solo. Esses dados confirmam os obtidos por Albuquerque et al. (2001), Imhoff et al. (2000) e Secco et al. (2004); ou seja, no plantio direto, o tráfego de máquinas e implementos causa o aumento da resistência do solo.

Nos tratamentos L1P3 e L4P4, a introdução de pastagem em 1999 e 2000 e a introdução de lavoura em sistema de plantio direto sobre pastagem em 2002, independente da profundidade, não causaram alterações na resistência do solo. Esse fato indica que a implantação de plantio direto de lavoura sobre pastagem e, ou, plantio direto de pastagem condiciona melhor qualidade física do solo às culturas.
No tratamento LCPD, houve aumento significativo da $\mathrm{RP}$ até a profundidade de $0,25 \mathrm{~m}$. A resistência do solo passou a ser significativa a partir do $5^{\circ}$ ano de plantio direto, nas camadas 0,05 e $0,10 \mathrm{~m}$; no $6^{\circ}$ ano, para a camada $0,15 \mathrm{~m}$; no $8^{\circ}$ ano, para $0,20 \mathrm{~m}$; e no $12^{\circ}$ ano, para $0,25 \mathrm{~m}$. Esses resultados sugerem que o efeito do tráfego de máquinas e implementos sobre a superfície do solo, com o passar do tempo, foi transmitido para as camadas mais profundas. Os sistemas de manejo LCCD e L1P3 podem estar comprometendo o crescimento do sistema radicular das plantas, por apresentarem resistência do solo acima de 2,0 MPa, valor considerado crítico, segundo Taylor et al. (1966), Imhoff et al. (2001) e Reichert et al. (2004).

Na figura 2, observa-se a relação à resistência à penetração e densidade do solo. Verifica-se que a resistência do solo foi positivamente relacionada com a densidade nos sistemas de manejo L1P3, L4P4 e LCPD, em concordância com o trabalho de Albuquerque et al. (2001). Para os tratamentos CERR 
Quadro 4. Resistência do solo à penetração de diferentes sistemas de manejo, em razão dos anos e das profundidades

\begin{tabular}{|c|c|c|c|c|c|c|c|c|c|}
\hline \multirow{2}{*}{ Trat. } & \multirow{2}{*}{1996} & \multirow{2}{*}{1997} & \multirow{2}{*}{1999} & \multirow{2}{*}{2000} & \multirow{2}{*}{2002} & \multirow{2}{*}{2006} & \multirow{2}{*}{ Média } & \multicolumn{2}{|c|}{ CV (\%) } \\
\hline & & & & & & & & $\mathbf{a}$ & b \\
\hline & \multicolumn{7}{|c|}{$-\mathrm{MPa}-$} & \multicolumn{2}{|c|}{$-\%-$} \\
\hline \multicolumn{10}{|c|}{$0,05 \mathrm{~m}$} \\
\hline CERR & $1,52 \mathrm{a}$ & $1,47 \mathrm{a}$ & $1,36 \mathrm{a}$ & $1,31 \mathrm{a}$ & $1,32 \mathrm{a}$ & $1,44 \mathrm{a}$ & 1,40 & 27,90 & 25,80 \\
\hline PCCA & $1,91 \mathrm{a}$ & $1,93 \mathrm{a}$ & $1,94 \mathrm{a}$ & $1,99 \mathrm{a}$ & $2,00 \mathrm{a}$ & $2,03 \mathrm{a}$ & 1,97 & & \\
\hline L1P3 & $1,95 \mathrm{~b}$ & $2,05 \mathrm{ab}$ & $2,11 \mathrm{ab}$ & $2,14 \mathrm{a}$ & $2,21 \mathrm{a}$ & $2,23 \mathrm{a}$ & 2,12 & & \\
\hline $\mathrm{L} 4 \mathrm{P} 4$ & $1,08 \mathrm{~d}$ & $1,31 \mathrm{~d}$ & $2,21 \mathrm{ab}$ & $2,05 \mathrm{bc}$ & $1,98 \mathrm{c}$ & $2,25 \mathrm{a}$ & 1,81 & & \\
\hline LCPD & $1,76 \mathrm{~b}$ & $1,96 \mathrm{~b}$ & $2,14 \mathrm{ab}$ & $\begin{array}{c}2,16 \mathrm{a} \\
0,10 \mathrm{~m}\end{array}$ & $2,17 \mathrm{a}$ & $2,21 \mathrm{a}$ & 2,07 & & \\
\hline CERR & $1,59 \mathrm{a}$ & $1,56 \mathrm{a}$ & $1,37 \mathrm{a}$ & $1,32 \mathrm{~b}$ & $1,37 \mathrm{a}$ & $1,34 \mathrm{a}$ & 1,43 & 25,61 & 21,09 \\
\hline PCCA & $1,92 \mathrm{a}$ & $1,94 \mathrm{a}$ & $1,92 \mathrm{a}$ & $1,95 \mathrm{a}$ & $1,96 \mathrm{a}$ & $1,97 \mathrm{a}$ & 1,94 & & \\
\hline L1P3 & $1,86 \mathrm{~b}$ & $2,10 \mathrm{a}$ & $2,08 \mathrm{a}$ & $2,04 \mathrm{a}$ & $2,16 \mathrm{a}$ & $2,08 \mathrm{a}$ & 2,05 & & \\
\hline L4P4 & $1,15 \mathrm{~d}$ & $1,41 \mathrm{c}$ & $2,10 \mathrm{a}$ & $2,00 \mathrm{a}$ & $1,71 \mathrm{~b}$ & $2,06 \mathrm{a}$ & 1,74 & & \\
\hline LCPD & $1,64 \mathrm{c}$ & $1,86 \mathrm{~b}$ & $2,05 \mathrm{a}$ & $\begin{array}{c}2,12 \mathrm{a} \\
0,15 \mathrm{~m}\end{array}$ & $2,16 \mathrm{a}$ & $2,18 \mathrm{a}$ & 2,00 & & \\
\hline CERR & $1,52 \mathrm{ab}$ & $1,65 \mathrm{a}$ & $1,37 \mathrm{ab}$ & $1,35 \mathrm{~b}$ & $1,42 a b$ & $1,41 \mathrm{ab}$ & 1,46 & 24,19 & 20,00 \\
\hline PCCA & 1,95 a & $1,95 \mathrm{a}$ & $1,93 \mathrm{a}$ & $1,94 \mathrm{a}$ & $1,91 \mathrm{a}$ & $1,90 \mathrm{a}$ & 1,93 & & \\
\hline L1P3 & $2,02 \mathrm{~b}$ & $2,19 \mathrm{ab}$ & $2,21 \mathrm{ab}$ & $2,23 \mathrm{a}$ & $2,22 \mathrm{a}$ & $2,25 \mathrm{a}$ & 2,19 & & \\
\hline $\mathrm{L} 4 \mathrm{P} 4$ & $1,22 \mathrm{~d}$ & $1,51 \mathrm{c}$ & $2,17 \mathrm{a}$ & $1,94 \mathrm{~b}$ & $1,73 \mathrm{c}$ & $2,04 \mathrm{ab}$ & 1,77 & & \\
\hline LCPD & $1,74 \mathrm{~b}$ & $1,83 \mathrm{~b}$ & $1,96 \mathrm{~b}$ & $\begin{array}{l}2,05 \mathrm{ab} \\
0,20 \mathrm{~m}\end{array}$ & $2,15 \mathrm{a}$ & $2,23 \mathrm{a}$ & 1,99 & & \\
\hline CERR & $1,64 \mathrm{a}$ & $1,65 \mathrm{a}$ & $1,58 \mathrm{a}$ & $1,53 \mathrm{a}$ & $1,57 \mathrm{a}$ & $1,66 \mathrm{a}$ & 1,61 & 27,46 & 19,52 \\
\hline PCCA & $1,89 \mathrm{a}$ & $1,85 \mathrm{a}$ & $1,87 \mathrm{a}$ & $1,83 \mathrm{a}$ & $1,88 \mathrm{a}$ & $1,87 \mathrm{a}$ & 1,86 & & \\
\hline L1P3 & $2,19 \mathrm{a}$ & $2,21 \mathrm{a}$ & $2,22 \mathrm{a}$ & $2,19 \mathrm{a}$ & $2,22 \mathrm{a}$ & $2,24 \mathrm{a}$ & 2,21 & & \\
\hline L4P4 & $1,29 \mathrm{c}$ & $1,62 \mathrm{c}$ & $2,24 \mathrm{a}$ & $1,89 \mathrm{~b}$ & $1,75 \mathrm{~b}$ & $1,83 \mathrm{~b}$ & 1,77 & & \\
\hline LCPD & $1,85 \mathrm{~b}$ & $1,80 \mathrm{~b}$ & $1,87 \mathrm{~b}$ & $\begin{array}{r}1,86 \mathrm{~b} \\
0,25 \mathrm{~m}\end{array}$ & $2,14 \mathrm{a}$ & $2,24 \mathrm{a}$ & 1,96 & & \\
\hline CERR & $1,86 \mathrm{a}$ & $1,83 \mathrm{a}$ & $1,79 \mathrm{a}$ & $1,74 \mathrm{a}$ & $1,72 \mathrm{a}$ & $1,93 \mathrm{a}$ & 1,81 & 23,58 & 19,70 \\
\hline PCCA & $1,91 \mathrm{a}$ & $1,94 \mathrm{a}$ & $1,92 \mathrm{a}$ & $1,92 \mathrm{a}$ & $1,97 \mathrm{a}$ & $1,91 \mathrm{a}$ & 1,93 & & \\
\hline L1P3 & $1,89 \mathrm{a}$ & $1,91 \mathrm{a}$ & $1,92 \mathrm{a}$ & $1,89 \mathrm{a}$ & $1,90 \mathrm{a}$ & $1,93 \mathrm{a}$ & 1,91 & & \\
\hline L4P4 & $1,36 \mathrm{c}$ & $1,72 \mathrm{~b}$ & $2,31 \mathrm{a}$ & $1,84 \mathrm{~b}$ & $1,78 \mathrm{~b}$ & $1,80 \mathrm{~b}$ & 1,80 & & \\
\hline LCPD & $1,73 \mathrm{c}$ & $1,77 \mathrm{c}$ & $1,79 \mathrm{bc}$ & $\begin{array}{l}1,89 \mathrm{bc} \\
0,30 \mathrm{~m}\end{array}$ & $1,98 \mathrm{~b}$ & $2,18 \mathrm{a}$ & 1,89 & & \\
\hline CERR & $1,65 \mathrm{a}$ & $1,63 \mathrm{a}$ & $1,69 \mathrm{a}$ & $1,64 \mathrm{a}$ & $1,62 \mathrm{a}$ & $1,63 \mathrm{a}$ & 1,64 & 26,83 & 19,48 \\
\hline PCCA & $1,63 \mathrm{a}$ & $1,62 \mathrm{a}$ & $1,68 \mathrm{a}$ & $1,68 \mathrm{a}$ & $1,69 \mathrm{a}$ & $1,65 \mathrm{a}$ & 1,66 & & \\
\hline L1P3 & $1,75 \mathrm{a}$ & $1,72 \mathrm{a}$ & $1,71 \mathrm{a}$ & $1,73 \mathrm{a}$ & $1,70 \mathrm{a}$ & $1,72 \mathrm{a}$ & 1,72 & & \\
\hline L4P4 & $1,93 \mathrm{a}$ & $1,92 \mathrm{a}$ & $1,94 \mathrm{a}$ & $1,81 \mathrm{a}$ & $1,87 \mathrm{a}$ & $1,93 \mathrm{a}$ & 1,90 & & \\
\hline LCPD & $1,93 \mathrm{a}$ & $1,94 \mathrm{a}$ & $1,96 \mathrm{a}$ & $1,98 \mathrm{a}$ & $1,95 \mathrm{a}$ & $1,97 \mathrm{a}$ & 1,96 & & \\
\hline
\end{tabular}

Trat.: tratamento; CERR: solo com vegetação natural; PCCA: solo com pastagem contínua e adubada; L1P3: solo sob lavoura de soja por um ano e por três anos sob pastagem de Brachiaria brizantha; L4P4: solo sob lavoura por quatro anos e por quatro anos sob pastagem de Panicum maximum; e LCPD: solo sob lavoura de soja em sistema de plantio direto. CV: coeficiente de variação, (a) subparcelas anos e (b) parcelas (Tratamento). Letras iguais na linha não diferem entre si pelo teste de Tukey a $5 \%$.

e PCCA, não foi constatada dependência significativa da $\mathrm{RP}$ em relação à Ds (Figura 2), provavelmente associada à maior macroporosidade, resultante da menor degradação da estrutura do solo e maior atividade radicular, que mantêm uma bioporosidade mais contínua e estável sob esses sistemas de manejo. Outros autores também verificaram comportamento semelhante como pode ser observado nos trabalhos de Bayer \& Mielniczuk (1999) e Salton et al. (2005).

\section{Estabilidades dos agregados em água}

No quadro 5, encontram-se os valores médios da porcentagem de agregados entre 8 e $2 \mathrm{~mm}$ nas camadas $0,0-0,10$ e $0,10-0,20 \mathrm{~m}$. Verificou-se efeito significativo da interação tratamento e ano $(p>0,01)$, para as duas profundidades estudadas (Quadro 5). O desdobramento da interação seguiu o mesmo procedimento realizado para densidade do solo e resistência do solo à penetração. 


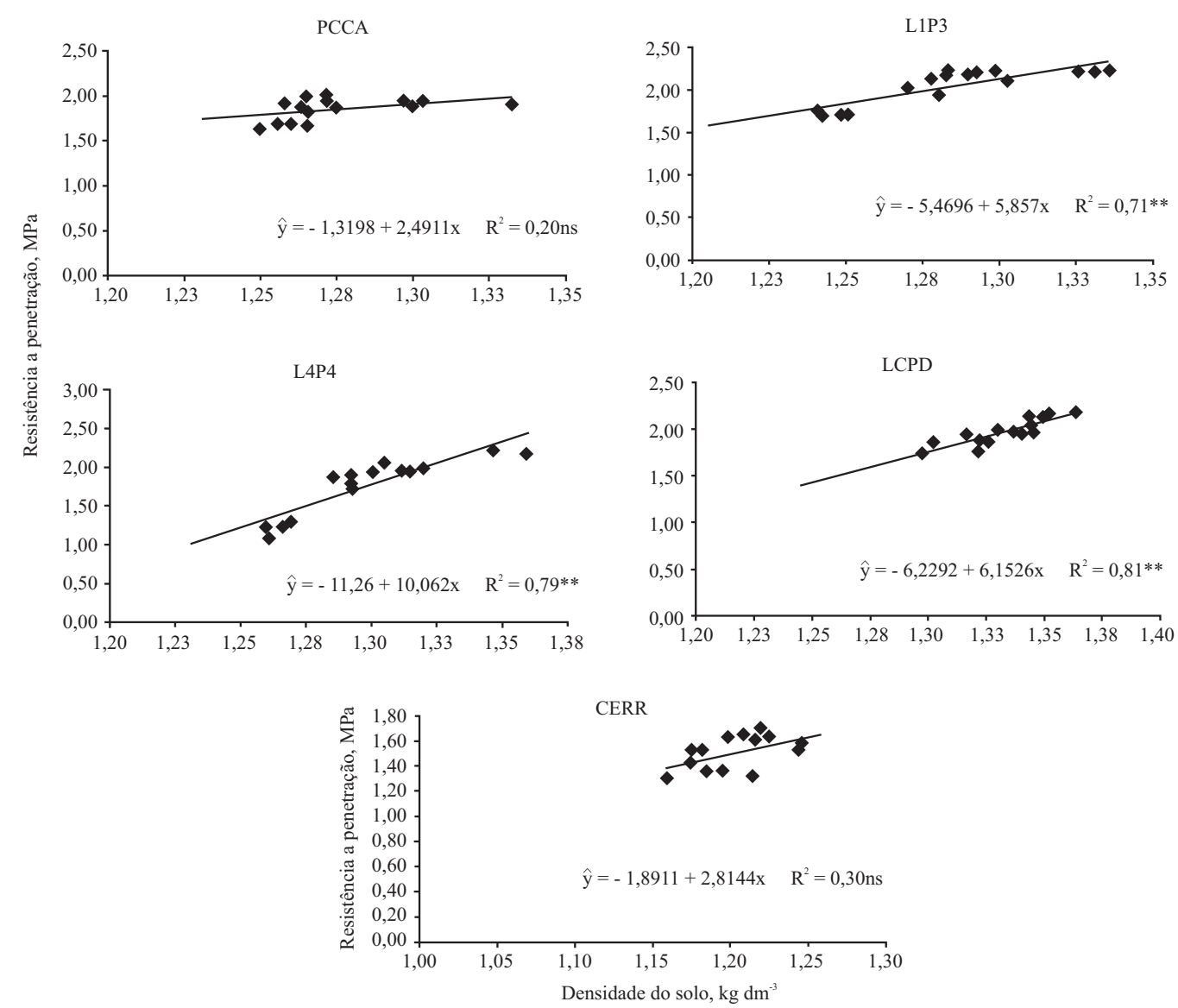

Figura 2. Relação entre a resistência do solo à penetração com a densidade do solo em diferentes sistemas de manejo no Cerrado. ns: não significativo, * $\mathbf{e}^{* *}$ significativo a 5 e $1 \%$, respectivamente. CERR: solo com vegetação natural; PCCA: solo com pastagem contínua e adubada; L1P3: solo sob lavoura de soja por um ano e por três anos com pastagem de Brachiaria brizantha; L4P4: solo sob lavoura por quatro anos e por quatro anos com pastagem de Panicum maximum, e LCPD: solo com lavoura de soja em sistema de plantio direto.

Quadro 5. Agregados estáveis entre 8 e 2 mm de diferentes sistemas de manejo do solo em razão dos anos e das profundidades

\begin{tabular}{|c|c|c|c|c|c|c|}
\hline \multirow{2}{*}{ Tratamento } & \multirow{2}{*}{1995} & \multirow{2}{*}{2000} & \multirow{2}{*}{2006} & \multirow{2}{*}{ Média } & \multicolumn{2}{|c|}{ CV } \\
\hline & & & & & $\mathbf{a}$ & $\mathbf{b}$ \\
\hline \multicolumn{7}{|c|}{$0,0-0,10 \mathrm{~m}$} \\
\hline CERR & $42,0 \mathrm{a}$ & $46,4 \mathrm{a}$ & $45,0 \mathrm{a}$ & 44,5 & & \\
\hline PCCA & $23,7 \mathrm{c}$ & $47,8 \mathrm{~b}$ & 63,8 a & 45,1 & & \\
\hline L1P3 & $25,9 \mathrm{~b}$ & $31,1 \mathrm{a}$ & $32,2 \mathrm{a}$ & 29,7 & 18,5 & 15,6 \\
\hline L4P4 & $8,6 \mathrm{c}$ & $19,3 \mathrm{~b}$ & $29,4 \mathrm{a}$ & 19,1 & & \\
\hline LCPD & $5,7 \mathrm{~b}$ & $8,5 \mathrm{~b}$ & 17,9 a & 10,7 & & \\
\hline \multicolumn{7}{|c|}{$0,10-0,20 \mathrm{~m}$} \\
\hline CERR & 32,9 a & $35,5 \mathrm{a}$ & $30,2 \mathrm{a}$ & 32,9 & & \\
\hline PCCA & $22,4 \mathrm{c}$ & $44,7 \mathrm{~b}$ & 58,4 a & 41,8 & & \\
\hline L1P3 & $14,5 \mathrm{~b}$ & $28,7 \mathrm{a}$ & $19,3 \mathrm{ab}$ & 20,8 & 16,2 & 13,3 \\
\hline L4P4 & $7,4 \mathrm{~b}$ & $15,1 \mathrm{a}$ & $18,2 \mathrm{a}$ & 13,6 & & \\
\hline LCPD & $5,3 \mathrm{~b}$ & $9,6 \mathrm{ab}$ & $12,7 \mathrm{a}$ & 9,2 & & \\
\hline
\end{tabular}

CERR: solo com vegetação natural; PCCA: solo com pastagem contínua e adubada; L1P3: solo sob lavoura de soja por um ano e por três anos com pastagem de Brachiaria brizantha; L4P4: solo sob lavoura por quatro anos e por quatro anos com pastagem de Panicum maximum; e LCPD: solo com lavoura de soja em sistema de plantio direto. . CV: coeficiente de variação, (a) subparcelas (anos) e (b) parcelas (Tratamento). Letras iguais na linha não diferem entre si pelo teste de Tukey a $5 \%$. 
As menores porcentagens de agregados estáveis registradas em 1995, independentemente da profundidade em todos os tratamentos, com exceção do CERR, estão associadas ao preparo do solo no início do experimento, utilizando-se arado de aiveca de disco e grade niveladora e aradora.

O tratamento PCCA apresentou ao longo dos anos os maiores valores do percentual de agregados entre 8 e $2 \mathrm{~mm}$, na camada de $0-0,10 \mathrm{~m}$, superando os valores do solo com vegetação natural. Esses resultados discordam dos trabalhos de Ayarza et al. (1993) e Alvarenga \& Davide (1999), os quais relataram que solo cultivado com pastagem apresenta o mesmo percentual de agregados, em relação a solos naturais. Essa agregação no PCCA deve ser atribuída à fertilização do solo e ao manejo animal, que propicia intensa rebrota e consequentemente a renovação da biomassa radicular, que, associada à ausência de preparo do solo, contribui para a formação de agregados de maior tamanho (Corazza et al., 1999; Six et al., 2004; Marchão et al., 2007).

Entre os tratamentos estudados, o LCPD foi o que apresentou os menores valores de percentual de agregação, atribuídos ao estoque de carbono no solo, corroborando com os dados de Salton et al. (2005). No tratamento L4P4, a agregação do solo começou a ser significativa a partir de 2000. Esse fato é em razão do preparo convencional do solo com grade aradora e niveladora para plantio da soja até 1998. A fertilização durante quatro anos dessa cultura propiciou a intensa brotação e produção de biomassa radicular da forrageira $P$. maximum, o que refletiu na agregação.

\section{CONCLUSÕES}

1. Os sistemas de manejo que causaram menor impacto nas propriedades físicas do solo foram os de pastagem contínua de $B$. decumbens cv. Basilisk com adubação a cada dois anos, e a integração lavoura-pecuária com quatro anos de lavoura de soja e quatro anos de pastagem com $P$. maximum cv. Tanzânia.

2. O pisoteio animal (lotação até 1,6 UA) não influenciou a densidade do solo e a resistência do solo à penetração nas áreas sob manejo com pastagem de Brachiaria decumbens que receberam adubação de manutenção a cada dois anos.

3. A Brachiaria brizantha cv. Marandu não apresentou a mesma capacidade de manter a qualidade física do solo em relação às forrageiras Brachiaria decumbens cv. Basilisk com adubação de manutenção e Panicum maximum cv. Tanzânia, introduzida após quatro anos de soja.

\section{LITERATURA CITADA}

ALBUQUERQUE, J.A.; SANGOI, L. \& ENDER, M. Efeitos da integração lavoura-pecuária nas propriedades físicas do solo e características da cultura do milho. R. Bras. Ci. Solo, 25:717-723, 2001

ALVARENGA, M.I.M. \& DAVIDE, A.V. Características físicas e químicas de um Latossolo Vermelho-Escuro e a sustentabilidade de agroecossistemas. R. Bras. Ci. Solo, 23:933-942, 1999.

ALVES, M.C.; SUZUKI, L.A.S. \& SUZUKI, L.E.A.S. Densidade do solo e infiltração de água como indicadores da qualidade física de um Latossolo Vermelho distrófico em recuperação, R. Bras. Ci. Solo, 31:617-625, 2007.

ANDREWS, S.S.; KARLEN, D.L. \& MITCHELL, J.P. A comparison of soil quality indexing methods for vegetable production systems in Northern California. Agric. Ecosyst. Environ., 90:25-45, 2002.

AYARZA, M.; VILELA, L. \& RAUSCHER, F. Rotação de culturas e pastagens em um solo de Cerrado: Estudo de caso. In: CONGRESSO BRASILEIRO DE CIÊNCIA DO SOLO, 24., Goiânia, 1993. Cerrados: Fronteira agrícola do século XXI: Resumos... Goiânia, SBCS, 1993. v.3. p.121122.

BAYER, C. \& MIELNICZNUK, J. Dinâmica e função da matéria orgânica In: SANTOS, G.A. \& CAMARGO, F.A., eds. Fundamentos da matéria orgânica do solo: Ecossistemas tropicais e subtropicais. Porto Alegre, Gênesis, 1999. p.9-26.

BUSSCHER, W.J.; BAUER, P.J.; CAMP, C.R. \& SOJKA, R.E. Correction of cone index for water content differences in a coastal plain soil. Soil Till. Res., 43:205-217, 1997.

CORAZZA, E.J.; SILVA, J.E.; RESCK, D.V.S. \& GOMES, A.C. Comportamento de diferentes sistemas de manejo como fonte ou depósito de carbono em relação à vegetação de Cerrado. R. Bras. Ci. Solo, 23:425-432, 1999.

COSTA, F.S.; ALBUQUERQUE, J.A.; BAYER, C.; FONTOURA, S.M.V. \& WOBETO, C. Propriedades físicas de um Latossolo Bruno afetadas pelos sistemas plantio direto e preparo convencional. R. Bras. Ci. Solo, 27:527$535,2002$.

EMPRESA BRASILEIRA DE PESQUISA AGROPECUÁRIA, Centro Nacional de Pesquisa de Soja, Tecnologias de produção de soja - região central do Brasil -2004, Londrina: Embrapa-soja, Embrapa-Cerrado, EmbrapaOeste, EPAMIG, Fundação Triângulo Mineiro, 237p. 2004.

EMPRESA BRASILEIRA DE PESQUISA AGROPECUÁRIA EMBRAPA. Centro Nacional de Pesquisa de solos. Manual de métodos de análise do solo. 2.ed. Rio de Janeiro, 1997. 212 .

EMPRESA BRASILEIRA DE PESQUISA AGROPECUÁRIA EMBRAPA. Manual de análises química de solos, plantas e fertilizantes. Brasília, Embrapa Comunicação para Transferência de Tecnologias, 1999. 370p.

FASSBENDER, W.H.W. \& BORNEMIZA, E. Química de suelos: Com enfasis em suelos de America Latina. 2.ed. San José, IICA, 1994. 420p. 
FERRI, M.G. Ecologia dos Cerrados. In: FERRI, M.G., coord. SIMPÓSIO SOBRE O CERRADO, BASES PARA UTILIZAÇÃO AGROPECUÁRIA, 4. São Paulo, 1977. Anais...São Paulo, Itatiaia, 1977. p.15-33.

GUEDES, H.M.; RESCK, M.V.S.; PERREIRA, I.S.; SILVA, J.E. \& CASTRO, L.H.R. Caracterização da distribuição do tamanho de agregados de diferentes sistemas de manejo e seu conteúdo de carbono em Latossolo Vermelho-Escuro na região dos Cerrados, Brasil. In: SIMPÓSIO SOBRE O CERRADO, 7., Brasília, 1996; INTERNATIONAL SYMPOSIUM ON TROPICAL SAVANNAS, 1, Brasília, 1996. Anais... Planaltina, Embrapa-CPAC, 1996. p.329333.

HAKANSSON, I. \& MEDVEDEV, V.W. Protection of soil from mechanical overloading by establishing limits for stresses caused by heavy vehicles. Soil Till. Res., 35:85-97, 1995.

HUSSAIN, I.; OLSON, K.R.; WANDER, M.A. \& KARLEN, D.C. Adaptation of soil quality indices on application to three tillage systems in southern Illinois. Soil Till. Res., 50:237249, 1997.

IMHOFF, S.; SILVA, A.P.; DIAS JÚNIOR, M.S. \& TORMENA, C.A. Quantificação de pressões críticas para o crescimento de plantas. R. Bras. Ci. Solo, 25:11-18, 2001.

IMHOFF, S.; SILVA, A.P. \& TORMENA, C.A. Aplicações da curva de resistência no controle da qualidade física de um solo sob pastagem. Pesq. Agropec. Bras., 35:1493-500, 2000.

LIMA, C.G.R.; CARVALHO, M.P., MELLO, L.M.M. \& LIMA, R.C. Correlação linear e espacial entre a produtividade de forragem, a porosidade total e a densidade do solo de Pereira Barreto (SP). R. Bras. Ci. Solo, 31:1233-1244, 2007.

KANNO, T.; MACEDO, M.C.M.; NAKAMURA, T.; YOSHIMURA, Y.; FUKUDA, E.; KANDA, K.; NAKANISHI, N.; SAITO, M. \& ANDO, Y. Grassland management techonology for sustainable Agro-pastoral systems in subtropical zone of Brazil. In: SUENAGA, K.; OSHIBE, A. \& TANIGUCHI, T., eds. Development of sustainable agro-pastoral systems in the subtropical zone of Brazil. Tsukuba, 2004. p.5-13. (JIRCAS Working Report, 36)

KAY, B.D. Rates of changes of soil structure under different cropping systems. Adv. Soil Sci., 12:1-51, 1990.

KAY, B.D.; RASIAH, V. \& PERFECT, E. Structural aspects of soil resiliency. In: GREENLAND, D.J. \& SZABOLCS, I., eds. Soil resilience and sustainable land use. London, CAB International, 1994. p.449-468.

KEMPER, W.D. \& CHEPIL W.S. Size distribution of aggregates. In: BLACK C.A., ed. Methods of soil analysis. Madison, America Science of Agronomy, 1965. v.9. p.499510 .

KLUTHCOUSKI, J. \& STONE, L.F. Manejo sustentável dos solos dos Cerrados. In: KLUTHCOUSKI, J.; STONE, L.F. \& AIDAR, H., eds. Integração lavoura-pecuária. Goiânia, Embrapa Arroz e Feijão, 2003. p.61-104.

KONDO, M.K. \& DIAS JUNIOR, M.S. Efeito do manejo e da umidade no comportamento compressivo de três Latossolos. R. Bras. Ci. Solo, 23:497-506, 1999.
LAL, R. Soil quality and sustainability. In: LAL, R.; BLUM, W.H.; VALENTINE, C. \& STEWRT, B.A., eds. Methods for assessment of soil degradations. New York, CRP Press, 1998. p.17-30.

MACEDO, M.C.M.; ZIMMER, A.H.; MIRANDA, C.H.B.; COSTA, F.P.; KANNO, T.; BONO, J.A.M. \& FUKUDA, E. Result of soybean yield. Animal liveweigth gain and soil fertility changes in agro-pastoral systems. In: SUENAGA, K.; OSHIBE, A. \& TANIGUCHI, T., eds. Development of sustainable agro-pastoral systems in the subtropical zone of Brazil. Tsukuba, 2004. p.15-18. (JIRCAS Working Report, 36)

MARCHÃO, R.L.; BALBINO, L.C.; SILVA, E.M.; SANTOS JUNIOR, J.D.G., SÁ, M.A.C.; VILELA, L. \& BECQUER, T. Qualidade física de um Latossolo Vermelho sob sistemas de integração lavoura-pecuária no Cerrado. Pesq. Agropec. Bras., 42:873-882, 2007.

PIMENTEL-GOMES, F. \& GARCIA, C.H. Estatística aplicada a experimentos agronômicos e florestais. Piracicaba, FEALQ, 2002. 309p.

PINZÓN, A. \& AMEZQUITA, E. Compactación de suelos por el pisoteo de animales en pastoreo en el piedmonte amazónico de Columbia. Past. Trop., 3:21-26, 1991.

REICHERT, J.M.; REINERT, J.D.; SILVA, V.R. Compactação do solo em sistema de plantio direto: Limites críticos e mitigação, In: COUTO. E.G. \& BUENO, J.F., orgs. REUNIÃO BRASILEIRA DE MANEJO E CONSERVAÇÃO DO SOLO E DA ÁGUA, 14., Cuiabá, 2004. Anais... Cuiabá, SBCS/UFMT, 2004. p.167-198.

SALTON, J.C.; MIELNICZUK, J.; BAYER, C.; FABRÍCIO, A.C.; MACEDO, M.C.M.; BROCH, D.L.; BOENI, M. \& CONCEIÇÃO, P.C. Matéria orgânica do solo na integração Lavoura-Pecuária em Mato Grosso do Sul. Dourados, Embrapa Agropecuária Oeste, 2005. 58p. (Boletim de Pesquisa e Desenvolvimento, 29)

SANTOS, J.L.S.; CASTRO, L.M.; COSTA, A.R. \& BALBINO, L.C. Resistência a penetração do solo no sistema de integração lavoura pecuária em Cerrado. In: CONGRESSO BRASILEIRO DE CIÊNCIA DO SOLO, 30., Recife, 2005. Anais... Recife, Sociedade Brasileira de Ciência do Solo, 2005. CD ROM

SECCO, D.; REINERT, D.J.; REICHERT, J.M. \& DA ROS, C.O. Produtividade de soja e propriedades físicas de um Latossolo submetido a sistemas de manejo e compactação. R. Bras. Ci. Solo, 28:797-804, 2004.

SEGUY, L.; KLUTHCOUSKI, J.; SILVA, J.G.; BLUMENSCHEIN, F.N. \& DALLAGUA, F.M. Técnicas de preparo de solo: Efeitos na fertilidade do solo e na conservação do solo ervas daninhas e na conservação da água. Goiânia, Embrapa - CNPAF, 1984. p.20. (Circular Técnica, 17)

SHEATH, G.W. \& CARLSON, W.T. Impact of cattle treading on hill lend. 1. Soil damage patterns and pasture status. New Zealand J. Agric. Res., 41:271-278, 1998.

SILVA, I.F. \& MIELNICZUK, J. Avaliação do estado de agregação do solo afetado pelo uso agrícola. R. Bras. Ci. Solo, 21:313-319, 1997. 
SILVA, A.P.; IMHOFF, S.; TORMENA, C.A.; LIMA, C.R. \& TAHAMA, R.S. Qualidade física de solos sob sistemas intensivos de pastejo rotacionado. In: SIMPÓSIO SOBRE A PASTAGEM, 19., 2002, Piracicaba. Anais... Piracicaba, 2002. p.79-97.

SILVA, A.P.; TORMENA, C.A. \& IMHOFF, S. Intervalo hídrico ótimo. In: MORAES, M.H.; MULLER, M.M.L. \& FOLONI, J.S.S., eds. Qualidade física do solo. Jaboticabal, FUNEP, 2001. p.1-20.

SINGLETON, P.L. \& ADDISON, B. Effects of cattle treading on physical properties of three soils used for dairy farming in the Waikato, North Island, New Zealand Aust. J. Soil Res., 37:891-902, 1999.

SIX, J.; BOSSUYT, H.; DEGRYZE, S. \& DENEF, K. A history of research on the link between (micro) aggregates, soil biota, and soil organic matter dynamics. Soil Till. Res., 79:7-31, 2004.
SOUSA, A.R.; SILVA, A.B.; RESENDE, M. Influência da pressão exercida por pisoteio de animais na compactação do solo do vale do Pajeú, em Pernambuco. In: REUNIÃO BRASILEIRA DE MANEJO E CONSERVAÇÃO DO SOLO E DA ÁGUA, 12, 1998, Fortaleza, Anais ..., Fortaleza: SBCS, p:327, 1998.

TAYLOR, H.M.; ROBERSON, G.M. \& PARKER Jr, J.J. Soil strength-root penetration relations to medium to coarsetextured soil materials. Soil Sci., 102:18-22, 1966.

TORMENA, C.A.; ROLOFF, G. \& SÁ, J.C.M. Propriedades físicas do solo sob plantio direto influenciadas por calagem, preparo inicial e tráfego. R. Bras. Ci. Solo, 22:301-309, 1998.

VIVALDI, L. Análise de experimentos com dados retidos ao longo do tempo. In: CONGRESSSO BRASILEIRO DE CIÊNCIA DO SOLO, 27., Brasília, 1999. Minicurso 6... Viçosa, MG, SBCS-CPAC, 1999. 26p. 